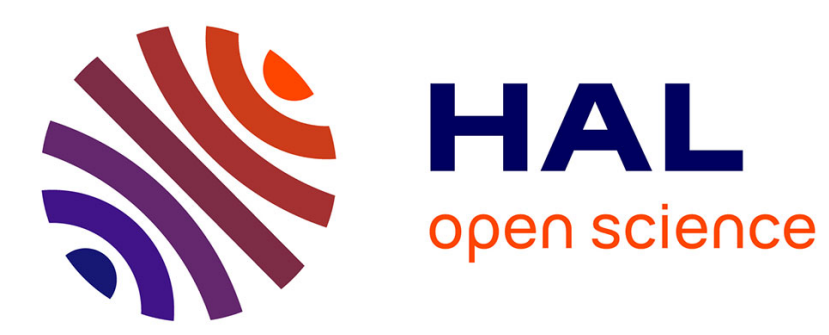

\title{
Les Plattenbauten et le déclin
}

\author{
Daniel Florentin
}

\section{To cite this version:}

Daniel Florentin. Les Plattenbauten et le déclin : "Effet Plattenbau ", politiques urbaines et représentations sociales dans les quartiers de grands ensembles à Leipzig. Géocarrefour - Revue de géographie de Lyon, 2011, Villes rétrécissantes en Allemagne, 86 (2). hal-01265935

\section{HAL Id: hal-01265935 \\ https://hal.science/hal-01265935}

Submitted on 2 Feb 2016

HAL is a multi-disciplinary open access archive for the deposit and dissemination of scientific research documents, whether they are published or not. The documents may come from teaching and research institutions in France or abroad, or from public or private research centers.
L'archive ouverte pluridisciplinaire HAL, est destinée au dépôt et à la diffusion de documents scientifiques de niveau recherche, publiés ou non, émanant des établissements d'enseignement et de recherche français ou étrangers, des laboratoires publics ou privés. 


\title{
Géocarrefour
}

Vol. 86/2 (2011)

Villes rétrécissantes en Allemagne

Daniel Florentin

\section{Les Plattenbauten et le déclin}

« Effet Plattenbau », politiques urbaines et représentations sociales dans les quartiers de grands ensembles à Leipzig

\author{
Avertissement \\ Le contenu de ce site relève de la législation française sur la propriété intellectuelle et est la propriété exclusive de \\ l'éditeur. \\ Les œuvres figurant sur ce site peuvent être consultées et reproduites sur un support papier ou numérique sous \\ réserve qu'elles soient strictement réservées à un usage soit personnel, soit scientifique ou pédagogique excluant \\ toute exploitation commerciale. La reproduction devra obligatoirement mentionner l'éditeur, le nom de la revue, \\ l'auteur et la référence du document. \\ Toute autre reproduction est interdite sauf accord préalable de l'éditeur, en dehors des cas prévus par la législation \\ en vigueur en France.
}

\section{revues.org}

Revues.org est un portail de revues en sciences humaines et sociales développé par le Cléo, Centre pour l'édition électronique ouverte (CNRS, EHESS, UP, UAPV).

\footnotetext{
Référence électronique

mars 2012, consulté le 02 janvier 2015. URL : http://geocarrefour.revues.org/8446

Éditeur : Association des amis de la Revue de Géographie de Lyon

http://geocarrefour.revues.org

http://www.revues.org

Document accessible en ligne sur : http://geocarrefour.revues.org/8446

Ce document est le fac-similé de l'édition papier.

(c) Géocarrefour
}

Daniel Florentin, «Les Plattenbauten et le déclin », Géocarrefour [En ligne], Vol. 86/2 | 2011, mis en ligne le 05 


\section{Daniel FLORENTIN}

Ecole Normale Supérieure, UMR

8504 Géographie-cités

\title{
Les Plattenbauten et le déclin
}

\section{" Effet Plattenbau n, politiques urbaines et représentations sociales dans les quartiers de grands ensembles à Leipzig}

\section{MOTS CLÉS}

Déclin urbain, Schrumpfung, Plattenbau, grands ensembles, Stadtumbau Ost, représentations, Leipzig

\section{KEY WORDS}

Urban shrinkage, Schrumpfung, Plattenbau, large housing estates, Stadtumbau Ost, social representation, Leipzig

\section{1 - Pour éviter toute} confusion, les termes de grands ensembles, de Platten ou de Plattenbauten seront employés sans distinction dans cet article. Strictement, les Plattenbauten recouvrent un aspect uniquement technique : ce sont des dalles en béton correspondant à des immeubles de 5, 11 ou 16 étages construits en série (Hannemann, 1995), alors que les grands ensembles sont associés dans l'imaginaire collectif aux quartiers d'habitat social.

2 - Dans leur article, M. Bernt et $\mathrm{S}$. Kabisch expliquent que les grands ensembles constituent de 5 à $75 \%$ du parc urbain dans les différents contextes européens, le taux de $75 \%$ étant atteint en Slovaquie.

\section{RÉSUMÉ}

Les grands ensembles de I'Est de l'Allemagne (Plattenbauten), souvent improprement considérés comme des logements sociaux sur le modèle ouest-allemand ou français, sont l'objet d'une représentation collective négative, idéologiquement et symboliquement marquée. Cette image a contribué à en faire une cible prioritaire des politiques de démolition dans le cadre de la politique de rénovation urbaine Stadtumbau Ost, concentrant des processus de déclin urbain sur certains quartiers, et créant de ce fait un " effet Plattenbau ". Cet effet entre cependant en contradiction avec la représentation de ces quartiers par ses habitants, ce qui est générateur de conflits.

\begin{abstract}
The large housing estates of East Germany, consisting of large, prefabricated concrete slabs (Plattenbauten), are often wrongly considered as social housing, as in the West German or French models. Collectively, these estates are perceived negatively, tainted both symbolically and ideologically. This image has contributed to making them a priority target for demolition in urban renovation policies (Stadtumbau Ost), resulting in the concentration of urban shrinkage in certain districts, creating a 'Plattenbau effect'. This effect contradicts, however, the more enthusiastic perception of these districts by their inhabitants, creating a potential source of social contention.
\end{abstract}

\section{INTRODUCTION}

" Die Platte ist besser als ihr Ruf " [Les grands ensembles valent mieux que leur réputation]. Ce titre presque provocateur d'un article de Tilo Arnhold (2005) reflète une double logique. II révèle en creux, d'un côté, la mauvaise réputation chronique des grands ensembles ${ }^{1}$ dans certains imaginaires collectifs, même si le grand ensemble est une composante importante du paysage urbain européen (Bernt, Kabisch, 2006) ${ }^{2}$. II dit, de I'autre, une réalité sociale éloignée de l'image clichée du grand ensemble comme lieu de concentration de la misère. Pour Bernt et Kabisch (2006), on retrouverait là une division traditionnelle entre I'Ouest et l'Est européens, les grands ensembles de l'Ouest étant évoqués en termes de problèmes sociaux, d'exclusion et de new urban underclass, tandis que les discours publics sur les grands ensembles dans la partie orientale de l'Europe considéreraient les grands ensembles comme une forme d'habitat " normal », et ne les évoqueraient $q^{\prime} u^{\prime} e n$ termes de maintenance et d'entretien.

Pour autant, en quelques années, l'image des grands ensembles de l'Est de l'Allemagne, les Plattenbauten, est devenue la représentation iconique des processus de déclin urbain, à la fois figure du déclin dans les imaginaires collectifs et cible de nombreux discours publics. Derrière cette combinaison d'une forme de dépression démographique, de mouvements de désindustrialisation et de fragilisation urbaine qui forge le canevas du déclin urbain (Fol et Cunningham-Sabot, 2010), les grands ensembles sont devenus le lieu des crispations sociales et des spirales socio-économiques négatives. Le débat sur la Schrumpfung [le déclin urbain], né du monde de la pratique à la suite notamment du rapport Lehmann-Grube sur la situation problématique des logements vacants dans les nouveaux Länder (Lehmann-Grube, 2000 ; Glock et Häussermann, 2004 ; Florentin,
Fol et Roth, 2008), s'est ainsi rapidement vu détourner : comme le souligne la littérature spécialisée, la représentation dominante a vite opéré le raccourci du million de logements vides au million d'unités de logements de grands ensembles vacants (Haller, 2002 ; Bernt et al., 2005).

Cette tension relative aux grands ensembles s'explique en partie par le fait qu'ils sont l'objet, de la part d'un grand nombre d'acteurs, de discours publics hautement défavorables, suscités par le rôle historique et idéologique de ces bâtiments. Les Plattenbauten sont, à l'origine, à la fois un modèle technique et un modèle sociologique (Hannemann, 1995), à savoir à la fois I'archétype de la conception techniciste du progrès autour des principes fonctionnalistes et le lieu d'accomplissement supposé du projet social socialiste (Schmidt, 1959). Les Plattenbauten ont donc non seulement été un objet technologique mais aussi l'instrument d'une doctrine politique devant permettre l'accomplissement personnel dans toutes les dimensions de la vie collective. Les Plattenbauten sont donc associés à une forme d'idéologie dont le régime de la République fédérale allemande a essayé d'écraser les structures (Bafoil, 2006). Ils étaient cependant également un symbole de citoyenneté, permettant notamment I'accomplissement du droit au logement garanti pour chacun qu'établissait la constitution du régime socialiste (Hannemann, 1995). Leur haute teneur idéologique et leur association à l'époque socialiste en font donc souvent l'objet d'un opprobre public dans les discours de nombreux responsables politiques (Keller, 2007).

Au-delà de ces considérations idéologiques, les quartiers de grands ensembles sont-ils véritablement plus fortement marqués par les processus de déclin que les quartiers d'habitat ancien (Haller, 2002) ? La question est de savoir à quel moment I'image extérieure du quartier influe au point de se transformer en réalité sociale. Même s'il est 
impossible de quantifier l'influence des représentations externes négatives sur le déclin objectif de ces quartiers, on cherchera à analyser les interactions entre cette image négative et les processus de déclin, notamment à travers I'analyse de l'évolution qu'a connu le programme de rénovation urbaine Stadtumbau Ost.

En d'autres termes, on peut se demander s'il n'y a pas une sorte "d'effet Plattenbau ", que I'on pourrait comprendre comme une forme aggravée du déclin, propre aux quartiers de grands ensembles, amplifiée en particulier par la politique de rénovation urbaine enclenchée au début des années 2000, Stadtumbau Ost. Cet 'effet Plattenbau' serait à la fois un processus de détérioration des conditions socio-économiques et urbaines des quartiers de grands ensembles, et le résultat de ce processus. II serait à la fois le reflet mais aussi le moteur de la dévalorisation de l'image externe du quartier ainsi que de la dégradation des conditions de vie dans le quartier.

Cet article se propose de tester la réalité de cet " effet grand ensemble " dans la ville de Leipzig, qui concentre dans son parc immobilier près de $30 \%$ de grands ensembles et s'est engagée précocement dans les stratégies de gestion du déclin (Lütke-Dalrup, 2003 ; Florentin, 2008). La seconde ville saxonne fut traditionnellement moins industrielle que ses concurrentes Dresde et Chemnitz (ancienne Karl-Marx-Stadt) ou que Berlin-Est, avec seulement 100000 emplois industriels pour près de 600000 habitants au moment de la chute du Mur, et quelques réinstallations industrielles symboliques mais peu riches en emplois comme les usines Porsche et BMW (Kunze et Lenk, 2007). Si certains chercheurs considèrent que la situation démographique de la ville s'est peu ou prou stabilisée (Wiessner, 2007), cela s'est fait au prix d'une aggravation des disparités socio-spatiales, dont les effets pourraient se concentrer dans certaines zones comme les grands ensembles.

La réalité de cet " effet grand ensemble " est testée à un double prisme. D'un côté, une analyse quantitative d'indicateurs démographiques, socioéconomiques et urbains cherche à en établir l'inscription matérielle dans ces quartiers. De l'autre, on cherchera à confronter une partie des discours négatifs et des indicateurs quantitatifs de déclin à la construction sociale d'une 'image interne' positive des Plattenbauten (Kil, 2004 ; Steinführer et Kabisch, 2005 ; Steinführer, 2006). Autrement dit, il s'agit de détailler la construction des perceptions et pratiques sociales des habitants de ces quartiers, en réaction aux discours négatifs ambiants et aux éléments de déclin objectif. Cette étude des représentations sociales sera menée à partir de l'analyse d'un journal local et d'une quarantaine $d$ 'entretiens qualitatifs réalisés auprès des habitants des grands ensembles leipzigois ${ }^{3}$. Nous cherchons à démontrer que les Plattenbauten sont l'objet d'un très fort attachement résidentiel plutôt que d'une sorte d'assignation résidentielle, même si cet équilibre est remis en cause par la politique de rénovation urbaine de Stadtumbau Ost.

Les perceptions des habitants dans des conditions de déclin ont été peu analysées dans la littérature aussi bien universitaire que professionnelle (Brandstetter et al., 2005), hormis quelques études de cas à Weisswasser, Wolfen, Hoyerswerda et Berlin (Schulz, 2004 ; Bernt et al., 2005 ; Keller, 2007). II s'agit donc d'un champ de recherche encore peu exploré, vu à travers le prisme d'un des hauts lieux de la transformation post-socialiste, les Plattenbauten.

Nous reviendrons dans un premier temps sur les différentes spirales du déclin pour tester la réalité de 'I'effet Plattenbau' à Leipzig, et son aggravation par la politique de Stadtumbau Ost. Puis seront analysées les perceptions et pratiques territoriales des habitants des quartiers de grands ensembles, pour souligner l'importance de l'attachement résidentiel. Une troisième partie viendra nuancer ce tableau radieux d'une image interne positive, en pointant les défis non résolus pour ces quartiers, notamment en matière de vieillissement.

\section{UN EFFET PLATTENBAU ? LE DÉCLIN AU PRISME DES INDICATEURS SOCIO-ÉCONOMIQUES}

Deuxième ville de Saxe par sa population (autour de 500000 habitants), Leipzig a connu un déclin continu depuis les années 1960 (Nuissl et Rink, 2004), ce qui en fait une shrinking city, insérée dans un contexte général de déclin qu'il convient $d^{\prime}$ analyser pour ne pas se centrer sur les seuls quartiers sensibles, mais les mettre en perspective dans un contexte d'échelle plus large (Préteceille, 2004). Ce déclin a fait de Leipzig à la fois I'un des laboratoires d'un éventuel " effet Plattenbau ", mais aussi un des lieux privilégiés de la politique de rénovation urbaine Stadtumbau Ost. Les effets de cette politique ont même fait office de facteur supplémentaire de déclin dans certains des quartiers concernés.

\section{Leipzig et les quatre spirales du déclin : la ville perforée}

\section{la spirale démographique}

A l'image d'une partie importante de l'Europe, et en particulier de la partie orientale de I'Allemagne, Leipzig est inscrite dans une spirale de déclin démographique résumée dans le schéma suivant (fig. 1).

la spirale socio-économique : la transformation post-socialiste et ses conséquences

Les effets de cette dynamique démographique régressive sont amplifiés par une crise économique
3 - Cet article est le fruit de travaux de M1 et M2 (2008 et 2009), encadrés par Sylvie Fol et Frédéric Dufaux, ponctués par un stage de quatre mois dans une coopérative de logements à Leipzig, chez le bailleur Kontakt d'avril à juillet 2009. 
Figure 1: Le déclin démographique allemand

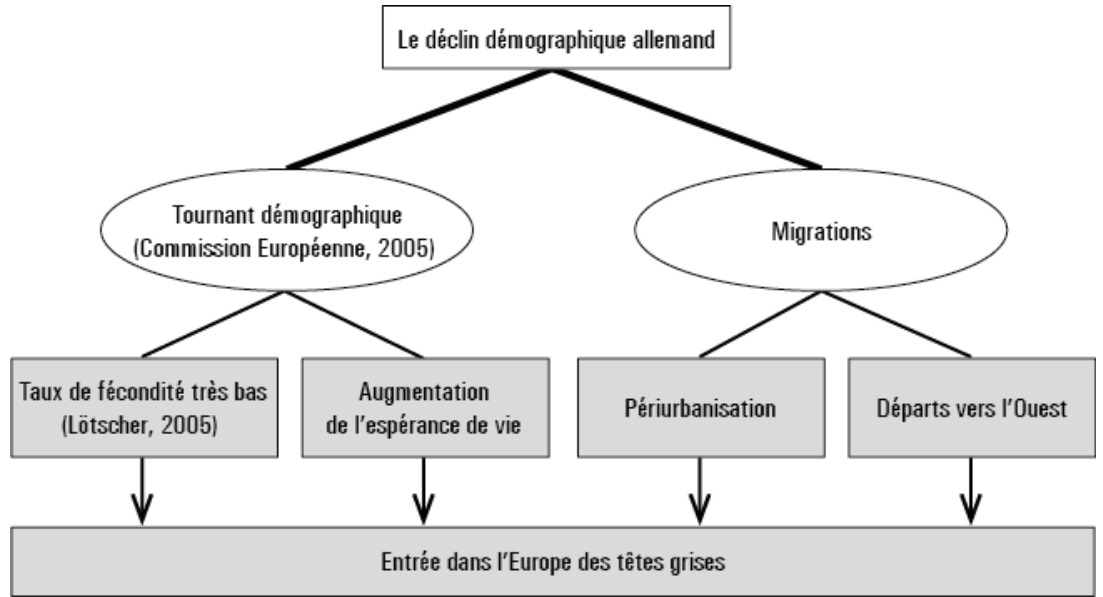

Leipzig de 1989 à 1999 (Nuissl \& Rink, 2004): la saignée démographique Tournant démographique : 25000 habitants en moins - Périurbanisation : 50000 habitants en moins Départs vers l'Ouest : 25000 habitants en moins - Déclin continu depuis 1962, pic de population en 1930 avec 700000 habitants et sociale profonde liée à la transformation postsocialiste. Plus qu'une simple mutation du tissu industriel, I'ensemble du bloc de l'Est et l'ancienne Allemagne de l'Est en particulier ont connu une profonde "thérapie de choc " (Bontje, 2004). Le processus a été si violent et si rapide par rapport à la désindustrialisation post-fordiste occidentale qu'il a été qualifié de "déséconomisation " par Christine Hannemann (2003). Derrière ce terme extrêmement fort, il faut comprendre une dissolution de la majeure partie du tissu productif, aussi bien agricole qu'industriel.

$C^{\prime}$ est tout le système productif et l'organisation du système économique qui se sont vus bouleversés en moins de dix ans. Face à une concurrence contre laquelle elle n'était pas armée, une bonne partie de l'industrie est-allemande, et en particulier leipzigoise, s'est retrouvée dans une situation de faillite presque immédiate. En à peine quatre années, de 1989 à 1993, la ville avait perdu 90000 de ses 100000 emplois industriels (Nuissl \& Rink, 2004), suivant notamment les recommandations de I'agence de tutelle Treuhandanstalt, qui s'est rapidement métamorphosée en agence de privatisation et de fermeture d'usines (Bafoil, 2006).

Les fermetures d'usines à Leipzig, et en particulier de la plupart des maisons d'édition qui faisaient le renom de la ville (comme Reklam, l'éditeur de livre de poche) ou des industries chimiques, se sont avant tout traduites par une montée en flèche des chiffres du chômage, qui flirtent encore aujourd'hui avec les 18 à $20 \%$, ce qui est le taux le plus élevé pour une ville de cette taille la moyenne nationale tournant autour de $10 \%$ (Kunze et Urbaniak, 2002 ; données de la ville de Leipzig). Là encore, Leipzig ne fait pas exception et s'insère profondément dans les cadres de ces processus d'érosion durable du tissu économique et urbain aggravés à l'issue de la chute du régime socialiste.

la spirale des finances publiques : des marges de mancuvre plus que réduites

A l'image de nombreuses communes allemandes, la ville de Leipzig connaît des difficultés finan- cières importantes (Bernt et Fritsche, 2008), et a vu ses finances publiques placées sous tutelle pendant quelques années pour opérer son désendettement de force (ACT, 2007). Elle est tiraillée par un effet de ciseaux entre des recettes fiscales limitées et des compétences toujours élargies (Guihéry \& Werner, 2005).

Comme les communes allemandes doivent notamment assumer la majeure partie des frais de l'aide sociale (aide au logement, allocations chômage), les deux premières spirales viennent contribuer à une fragilisation accrue des finances locales dans un contexte où l'intervention publique est sans doute plus nécessaire qu'ailleurs. Les marges de manœuvre des pouvoirs publics en sont réduites $d$ 'autant, particulièrement pour ce qui a trait aux politiques de rénovation ou de réhabilitation du parc immobilier ou au renforcement de son attractivité par un travail sur les espaces publics.

\section{la spirale urbaine : la transformation paysagère par} la vacance, la ville perforée

L'accumulation des spirales précédentes est à I'origine de l'apparition massive de friches, urbaines ou industrielles, créant un paysage particulier, propre à la ville en déclin, et qui fut décrit par I'un des responsables de l'aménagement de la ville de Leipzig comme une "ville perforée " (LütkeDaldrup, 2003). En 2006, entre 14 et $16 \%$ du parc total était vacant dans la ville, un des chiffres les plus élevés d'Allemagne pour une ville de ce rang.

L'ensemble de ces spirales font de Leipzig une ville en déclin, atteinte par des processus protéiformes mais cumulatifs, qui rendent la situation de déclin durable à l'échelle de la ville (fig. 2).

\section{L'effet Plattenbau : une réalité statistique ?}

A l'échelle infra-urbaine, ces dynamiques régressives s'expriment de façon différenciée, laissant la place à une ville aux mille visages, alternant entre des quartiers déclinants et parfois abandonnés ou délaissés par les pouvoirs publics ou les bailleurs, 


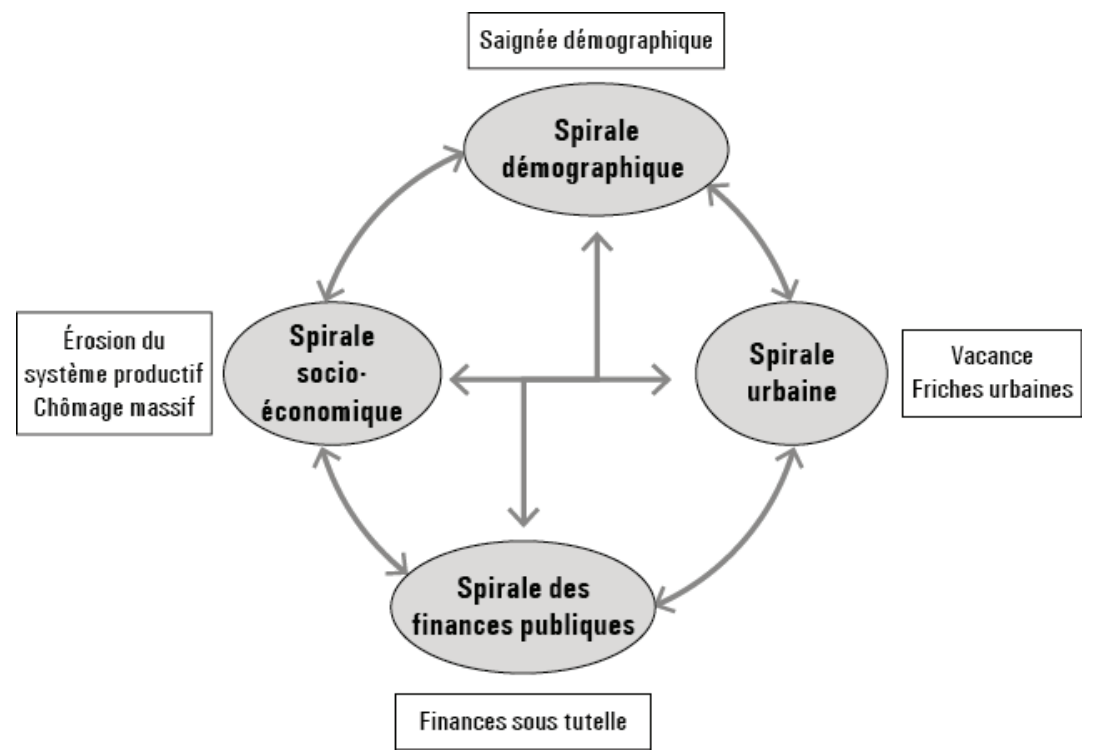

des quartiers en croissance dont on encourage le développement, et des quartiers stagnants dont le devenir est souvent vacillant. C'est à cette échelle infra-urbaine qu'on peut notamment identifier un éventuel 'effet Plattenbau'.

Trois blocs d'indicateurs quantitatifs ont ainsi servi à I'élaboration d'une classification ascendante hiérarchique ${ }^{4}$ (carte 1), dont le but est d'associer les quartiers selon leur profil pour faire émerger différents cas de déclin. Ces trois séries d'indicateurs cherchent à prendre en compte la diversité des facteurs du déclin urbain, et ont donc été isolés, en fonction des données disponibles et collectées par les services de la mairie de Leipzig :

- un bloc démographique (l'évolution de la population, les soldes migratoires et l'âge moyen),

- un bloc socio-économique (le taux de chômage, les bénéficiaires des minimas sociaux et les revenus moyens),

- et un bloc urbain (la vacance).

On constate que les quartiers proches du centre, d'habitat ancien de type wilhelminien (Gründerzeit), regroupés principalement dans le type 2 forment un ensemble caractérisé par de forts mouvements de dépopulation et un solde migratoire fortement négatif au début des années 1990, avant de voir la situation s'inverser au tournant des années 2000. Cette tendance confirme que les processus de déclin ont d'abord concerné les quartiers centraux, qui ont alors bénéficié de programmes de rénovation importants. Les spirales du déclin se sont en quelque sorte transférées vers les zones d'habitat de grand ensemble.

C'est autour des années 2000 également que les types 5 et 6 concentrent l'essentiel des spirales du déclin, avec une population tendanciellement fortement déclinante, un solde migratoire très en dessous de la moyenne, une population nettement plus âgée et vieillissant plus que la moyenne de la ville, et un taux de chômage relativement plus élevé que la moyenne. Autrement dit, ces profils sont ceux des quartiers les plus touchés par les phénomènes de déclin.

Le type 5 est même une sorte de modèle aggravé du type 6 , avec des tendances similaires, mais plus marquées. Ces profils correspondent notamment aux quartiers de Grünau, de Paunsdorf et de Mockau Nord. Ces quartiers recouvrent la majeure partie des territoires des grands ensembles, là où les Plattenbauten constituent l'écrasante majorité voire la totalité du parc. Apparaissent ainsi les caractéristiques d'un " effet Plattenbau ", avec une population vieille et vieillissante, socialement plus fragile, et d'où les habitants auraient tendance à partir. Au sein de cet effet Plattenbau, le cas de Grünau semble extrême, les quartiers du type 6 paraissant être affectés par ces spirales déclinantes avec un léger décalage temporel et une intensité moindre.

Si les premiers signes d'un effet Plattenbau semblent émerger, il faut sans doute nuancer sa profondeur et son expression spatiale dans l'attente de données plus complètes ${ }^{5}$. Le manque de continuité de certains indices rend leur pertinence moindre et limite l'apport des données quantitatives.

Pour autant, on peut retenir une détérioration des conditions démographiques, économiques et sociales au tournant des années 2000, concomitante des débuts du programme de rénovation urbaine Stadtumbau Ost, dont les grands ensembles ont été la cible principale.

Les quartiers de Plattenbauten comme lieu privilégié d'action de Stadtumbau Ost

Face à la situation inédite par son ampleur que représente le déclin urbain lié à la transformation post-socialiste, la sphère du monde immobilier a tôt réagi pour inciter les pouvoirs publics à mettre en place une politique publique répondant aux problèmes liés à la vacance et à la détente complète des marchés immobiliers. C'est à l'issue de la commission Lehmann-Grube (2000), rassemblant les principaux acteurs du monde opérationnel, qu'a ainsi été décidée la mise en place d'une politique de rénovation urbaine appelée Stadtumbau Ost, dont le financement s'établissait à 2,5 milliards d'euros sur la période 2002-2009 (Wiechmann, 2007). Le programme est non seulement financièrement ambitieux, mais aussi puissamment novateur, car, pour la première fois, il
Figure 2 : Les spirales du déclin à Leipzig

Carte 1 (ci-contre) :

Les quartiers de Leipzig selon une classification ascendante hiérarchique
4 - Pour obtenir des classes homogènes, c'est-à-dire minimiser les différences intraclasses et maximiser les différences interclasses, toutes les variables utilisées ont ainsi été normées, c'est-àdire centrées puis réduites.

5 - Les données dont nous disposions sont souvent trop récentes ou trop lacunaires pour mesurer la vigueur et les effets de la transformation post-socialiste. 

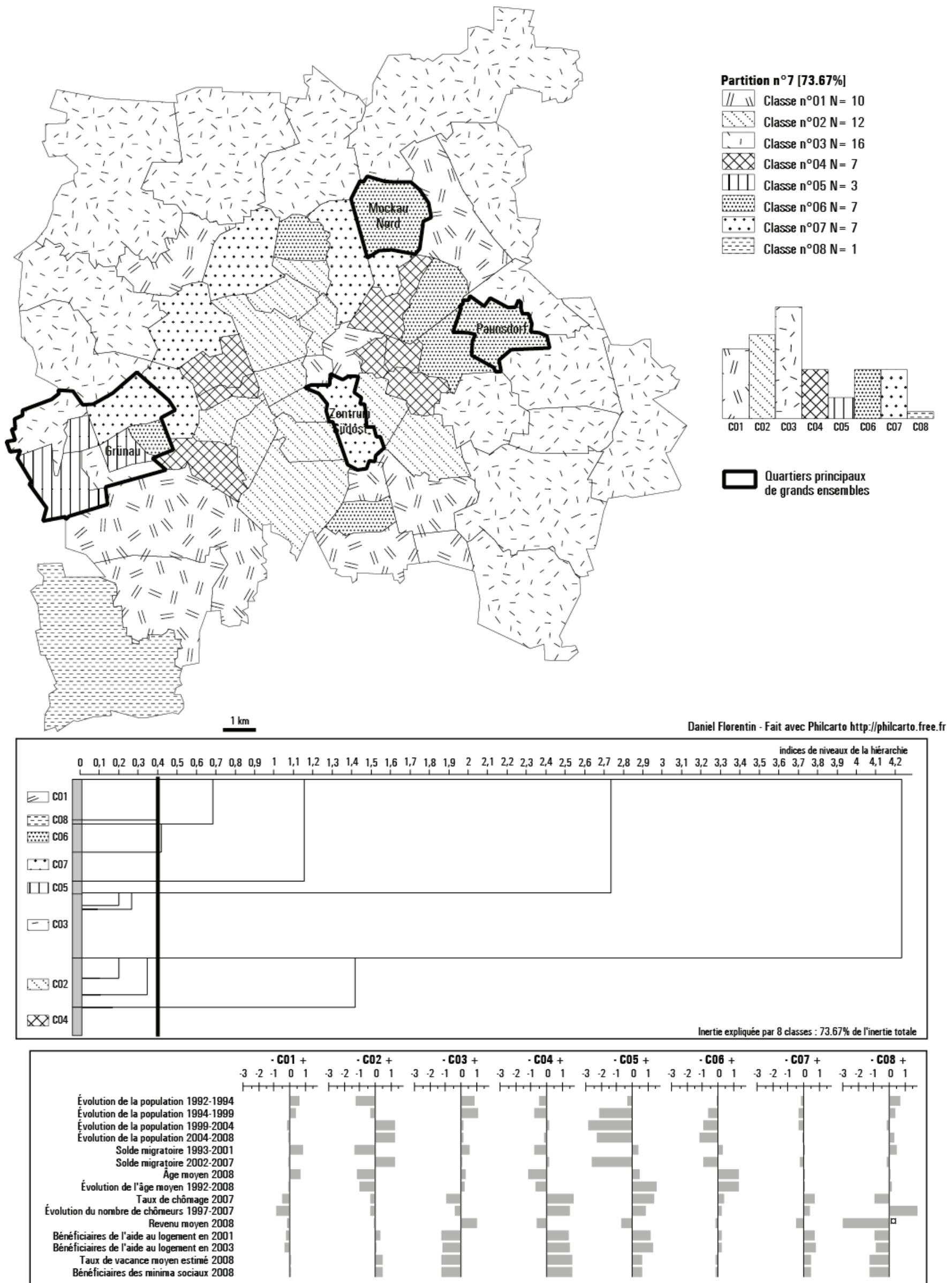

Chaque bâton représente la distance de la moyenne de chaque classe à la moyenne générale. Cette distance est exprimée en nombre d'écarts-types de chaque variable. à : bâton coupé à + ou - 3 écart-types 
prévoit la destruction sans reconstruction d'une part importante du parc immobilier dans la partie orientale de I'Allemagne. II opère ainsi un changement de paradigme majeur face à l'idéal traditionnel de la croissance (Bernt, 2009 ; Kil, 2001) ou aux expériences françaises comme celle de la rénovation urbaine et de son principe double de diversification de l'habitat et de " un pour un ", un logement reconstruit pour tout logement détruit (Noyé et Lelévrier, 2009).

Cependant, sa mise en pratique a été marquée par un certain nombre de détournements, ou par une interprétation assez fluctuante de l'esprit de la rénovation. On peut ainsi dénombrer trois principales dérives du programme.

\section{un ciblage quasi systématique et idéologique des grands ensembles}

La première est son ciblage quasi systématique des grands ensembles (ACT, 2007). Celui-ci s'explique par un double phénomène.

L'amalgame a souvent été fait dans les discours officiels et publics entre la baisse de population, la vacance, les Plattenbauten, et leur nécessaire démolition (Bernt, Kabisch, Peter, 2005). La concentration des démolitions sur les quartiers de grands ensembles relève même d'un projet politique porté par le Land, et en particulier par sa banque la Sächsische Aufbaubank, qui finance en grande partie la politique de rénovation, et impose ses vues aux différents bailleurs. Cette orientation de la banque a été particulièrement forte dans le cadre d'une politique de stricte démolition, présentée comme inéluctable, dans certains complexes d'habitation de Grünau, les Wohnkomplexe 7 et 8 (WK7 et WK8), regroupant plusieurs dizaines de milliers d'habitants. La banque de construction de Saxe a donc décidé à plus ou moins longue échéance de ne pas investir dans la revalorisation des quartiers de grands ensembles, au nom d'une logique économique qui cache plus sûrement des logiques politiques et idéologiques.

Par ailleurs, la politique de dégrèvements fiscaux visant à une participation large de tous les acteurs immobiliers $\mathrm{n}^{\prime} \mathrm{a}$ pas été suffisamment incitative pour pousser les propriétaires privés à participer au programme, et notamment aux démolitions (Glock et Häussermann, 2004 ; Bernt, 2003, 2005, 2008). C'est ce qui a conduit au phénomène classique de la théorie des jeux dit du dilemme du prisonnier : chacun attend que le voisin bouge avant de faire le moindre mouvement, et attend que l'un détruise pour avoir plus de chance de pouvoir louer ou vendre son bien (Bernt, 2009). Dans pareil contexte, seul l'acteur public, la Leipziger WohnungsBaugesellschaft (LWB), ainsi que les coopératives de logement (Wohnungsbaugenossenschaften) sont à même de pouvoir procéder aux démolitions, qui leur permettent de se désendetter fortement. La majeure partie de leur parc étant situé dans les Plattenbauten, les démolitions ont été opérées en grande majorité dans ce type de bâti, sans que les bâtiments concernés soient toujours vacants. En 2008, la LWB avait ainsi détruit 8300 logements $^{6}$, dont plus de 5500 dans les Plattenbauten, opérant par ce biais un retrait de fait de l'acteur public de certains quartiers de grands ensembles. Par la structure de son financement et des acteurs participant au programme, Stadtumbau Ost cible en fait indirectement les grands ensembles (encadré 1).

\section{Encadré 1 :}

"La banque $m$ 'a refusé un crédit pour un projet alternatif à la destruction complète, un projet de maisons-terrasses, car elle trouve que le quartien n'est pas assez sûr pour des investissements. Ce qui contribue finalement à ce que les projections pour Grünau soient mauvaises. Le directeur de la banque de Saxe m'a dit : Grünau, c'est une ventouse économique " (responsable coopérative de logement, 2009).

Les grands ensembles doivent donc supporter le fardeau de la remise à niveau du marché au nom du reste de la ville (Bernt, 2009).

une orientation quasi exclusive vers la démolition La deuxième dérive de Stadtumbau Ost est son orientation principale vers la démolition, au détriment de la revalorisation. Le financement de la démolition est assuré à 50\% par le Land, et à 50\% par la Fédération (Bund), alors que les mesures de revalorisation sont financées par tiers entre le Land, le Bund et la commune concernée. Le Land est malgré tout l'instance fixant la part accordée aux mesures de revalorisation. Le Land de Saxe, à travers son bras armé qu'est la Sächsische Aufbau Bank, a opté pour un équilibre plaçant $80 \%$ des investissements liés au programme dans la démolition, contre seulement $20 \%$ pour les mesures de revalorisation (Wiechmann \& Siedentop, 2005 ; Bernt et al., 2006), au motif que $\mathrm{d}^{\prime}$ autres programmes existaient déjà pour accomplir l'accompagnement social ou les projets d'embellissement, notamment avec le programme Soziale Stadt, pourtant financièrement moins doté (entretien responsable de la mairie de Leipzig). Cette option stratégique du Land de Saxe souligne un phénomène classique de découplage entre les politiques urbaines de transformation du bâti et les politiques sociales, et renforce indirectement les spirales du déclin.

conséquences : déstructuration du tissu urbain, image dégradée et insécurité locative

Comme les travaux de Matthias Bernt I'ont montré, la logique de Stadtumbau Ost manque ainsi singulièrement de planification d'ensemble : c'est
6 - Dont seulement 5000 étaient vacants. 
même une sorte de planification à trous qu'il décrit, reflet de l'hétérogénéité des acteurs et des intérêts en jeu, notamment le désendettement des bailleurs (Glock et Häussermann, 2004). Cette pratique de planification acte les décisions de destructions plus qu'elle ne les contrôle et les insère dans une logique urbaine plus générale (Bernt, 2003, 2004, 2005, 2008).

De façon concrète, la politique de rénovation urbaine, par ce ciblage, a aussi correspondu à une forme de déstructuration du tissu urbain dans ces quartiers. Celui-ci a pu prendre plusieurs formes. Les démolitions complètes opérées là où les bailleurs comptaient mettre en place des projets d'embellissement ont créé une frustration de la population. La démolition de certains services, comme des commerces de plain-pied, a amplifié l'impression d'une perforation de la trame urbaine.

Enfin, la politique de démolition s'est faite souvent sans grande transparence, au point qu'il a fallu mettre en place des garde-fous pour systématiser les procédures d'information et de relogement (Bernt et Fritsche, 2008). Elle a concerné un parc souvent en bon état (Kabisch et al., 2007), dont le coût d'entretien et de réparation est bien moindre que dans l'ancien. Le manque de transparence et le court délai de notification de démolition - un an dans le meilleur des cas - ont en fait entretenu un sentiment d'insécurité locative, qui a poussé bon nombre d'habitants au départ, amplifiant les dynamiques de dépopulation de certains quartiers de grands ensembles. Seuls $60 \%$ des locataires de la LWB concernés par les démolitions sont ainsi restés dans le parc du bailleur, signe d'une certaine défiance (entretien responsable LWB). La LWB a certes pu se séparer d'une partie de son parc vacant, et a vu ses chiffres de vacance passer de $20 \%$ avant le programme de rénovation à $4-6 \%$ en 2009 , mais elle l'a fait au prix d'une image dégradée, I'associant à la figure du bailleur-destructeur.

Les démolitions sans revalorisation contribuent à une dégradation de la réputation et ainsi de I'image du quartier, et donc à un renforcement des processus caractérisant le déclin urbain. Par ce ciblage systématique, on amplifie dans les Plattenbauten une spirale déjà à l'œuvre depuis le tournant des années 2000 (Bernt, 2009). Stadtumbau Ost est ainsi un agent d'amplification de 'I'effet Plattenbau'. C'est pour lutter contre l'aggravation de ces spirales que la plupart des bailleurs, et notamment la coopérative Kontakt, qui fut la seule à refuser la logique de démolition de Stadtumbau Ost, ont d'ailleurs signé un appel demandant I'arrêt des démolitions dans le quartier de Grünau dès 2006. Un tel appel montrait l'ampleur du malaise créé par les démolitions au sein de la population, en décalage avec l'image qu'elle se fait de son quartier.

\section{LES HABITANTS ET LA RÉNOVATION : CHRONIQUE D'UN ATTACHEMENT RÉSIDENTIEL PUISSANT}

Le raccourci traditionnel qu'on trouve dans les discours publics fait des zones de Plattenbauten à la fois la cause et la conséquence du déclin : la cause par un bâti souvent décrié, la conséquence par les mouvements de paupérisation dont elles seraient I'objet. En d'autres termes, ces grands ensembles, dans les représentations aussi bien des médias que de certains acteurs politiques, sont l'incarnation d'une forme d'assignation résidentielle, première étape vers une séparation sociale et spatiale plus importante.

A l'opposé de cette vision, les perceptions et pratiques sociales des habitants des grands ensembles, si elles se montrent sévères avec la politique de rénovation urbaine, témoignent aussi d'un phénomène inverse de l'assignation, celui d'un attachement résidentiel puissant (Kil, 2004 ; Bernt et Kabisch, 2006). Cet attachement est même nourri de l'image externe dégradée, et participe d'un mouvement de construction d'un discours interne visant à promouvoir une image différente des grands ensembles. S'est ainsi développée de manière endogène une image positive des grands ensembles qui cherche, de manière parfois volontariste, à contrer les dynamiques objectives du déclin et les représentations négatives associées aux grands ensembles.

Cette analyse repose sur deux vecteurs, une étude critique d'un journal local d'un quartier de grand ensemble et une série d'une quarantaine d'entretiens réalisés dans les quartiers de grands ensembles, principalement au sein du bailleur coopératif Kontakt.

Analyse des perceptions au prisme de la presse locale : le journal Grün As

Plutôt que d'étudier la perception extérieure des grands ensembles dans la presse nationale comme Germès et al. (2010), nous avons opté pour une approche plus endogène, partant $d^{\prime}$ un journal local du quartier emblématique de Grünau ${ }^{7}$, Grün As. Le but est d'identifier la représentation sociale interne du quartier.

Ce journal gratuit est né d'une insatisfaction portée par la Volkshochschule (sorte d'université populaire) dès 1995, pour réagir face au portrait toujours noir et négatif fait du quartier par les médias traditionnels. C'est donc pour contrebalancer cette vision que le journal a été créé quelques mois plus tard, et cherche à être un journal pour les habitants par les habitants ${ }^{8}$. Son succès ne se dément pas, puisqu'il tire entre 12000 et 15000 exemplaires par numéro. Le journal bénéficie ainsi d'un lectorat de 25000 à 30000 habitants, au- 
trement dit plus de la moitié de la population du quartier de Grünau, ce qui en fait un organe représentatif, et qui plus est apprécié par les habitants pour la qualité de son contenu.

Grün As cherche à promouvoir une image du quartier qui soit fidèle à celle que s'en font ses habitants. II se montre de ce point de vue assez critique de la politique de rénovation urbaine de Stadtumbau Ost, décrite comme un instrument de perforation économique, sociale et urbaine. Par ce biais, le journal est passé progressivement à une position critique ferme, cherchant explicitement à contrebalancer certains des aspects de "l'effet Plattenbau ".

\section{Quatre points font l'objet d'une cristallisation cri-} tique importante

La première crainte qui ressort des articles de Grün $A s$ consacrés à la rénovation urbaine est celle d'une relégation du quartier. Pour éviter cette relégation, le journal multiplie les reportages sur les rénovations réussies où les quartiers de grands ensembles sont placés sinon au centre du projet, du moins pas à sa marge, comme dans le quartier Clarenberg à Dortmund.

A cette crainte de la relégation s'associe une critique des rythmes et temporalités de la rénovation. On reproche à Stadtumbau Ost de procéder d'abord aux démolitions, au lieu d'opérer comme dans d'autres villes comme Leinefelde (Thuringe), où la rénovation urbaine a commencé par la valorisation (Aufwertung), suivie par la restructuration (Umbau) avant de procéder en toute fin aux démolitions (Abriss) (Grün As, Juillet 2001).

La critique se fait aussi plus matérielle et vigoureuse, face notamment aux projets de démolition de certains équipements, et notamment les bibliothèques. Chaque complexe d'habitation disposait à l'origine de sa bibliothèque, et la ville a prévu d'en fermer deux ou trois, pour " améliorer l'offre de services", comme le relate un des articles. Les journalistes et habitants se mobilisent pour démontrer, statistiques à l'appui, que la bibliothèque visée de Grünau Nord accueille chaque année plus de 65000 visiteurs. Une campagne se développe pour lutter contre le principe "Leipzig schliesst - statt Leizpig liest " (Leipzig ferme, plutôt que Leipzig lit !, titre d'un article de juillet 2003). Le journal se fait ici l'écho d'une perception générale de la population, celle d'un abandon progressif par les pouvoirs publics des quartiers de grands ensembles, alors que, dans le même temps, des sommes importantes sont déboursées pour des projets de rénovation dans le centre, ou pour des projets pharaoniques et hautement controversés comme le City Tunnel (Florentin, 2008).

Cet abandon notamment par la perte des services publics de base est ressenti de façon encore plus

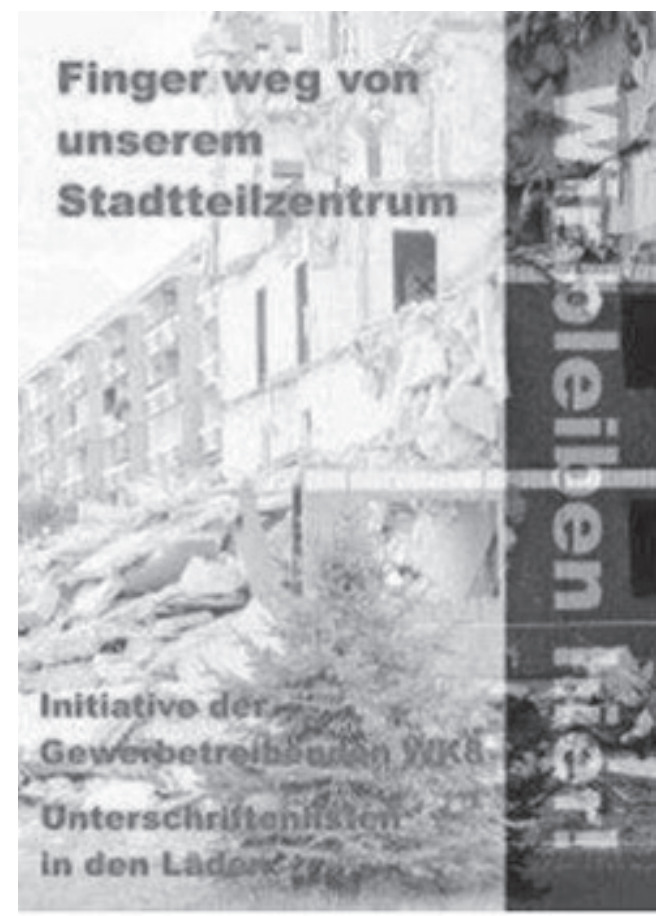

Photo 1 : Affiche « Finger weg von unserem Stadtteilzentrum " [touche pas à notre centre de quartier]

massive dans le cas déjà évoqué des opérations de rénovation/démolition. Le cas d'une tour de seize étages du bailleur communal, située sur la Brackestrasse, a cristallisé les tensions liées à la rénovation. Détruire la tour voulait dire raser les commerces de plain-pied, ce contre quoi la population s'est élevée $(2003 / 43 ; 2004 / 02)$. Les protestations commencent dès 2003, et le journal se fait l'un des porte-voix de la contestation, en incitant notamment à signer la pétition pour le maintien des commerces et à participer aux manifestations de protestation, derrière un slogan : "Finger weg von unserem Stadtteilzentrum " (touche pas à notre centre de quartier, photo 1). La tour sera finalement démolie et les commerces déplacés. L'ensemble a contribué à une détérioration de l'offre de services de proximité, dans un processus dont les habitants ont été exclus, et qui est ressenti par nombre d'entre eux comme une stratégie insidieuse de relégation des grands ensembles.

A la suite de cet épisode, le journal s'est fait l'écho et le point central du mouvement "Stadtumbau Grünau -so nicht! " (Stadtumbau à Grünau, pas de cette manière) (2006), qui appelle à un arrêt des démolitions, en particulier dans le WK8, et exige un nouveau plan de développement du quartier et une nouvelle politique de crédit de la Sächsische Aufbau Bank, qui finance le programme Stadtumbau Ost. Grün As démontre ainsi que le programme de rénovation, tel qu'il a été pratiqué, a davantage contribué à une dégradation de l'image et de la stabilité du quartier qu'à
7 - Deuxième plus grand quartier de grands ensembles de I'Est de I'Allemagne, construit à la fin des années 1970, fortement concerné par Stadtumbau Ost.

8 - Dans la pratique, la participation citoyenne à l'écriture du journal reste faible, par manque de volontaires. 


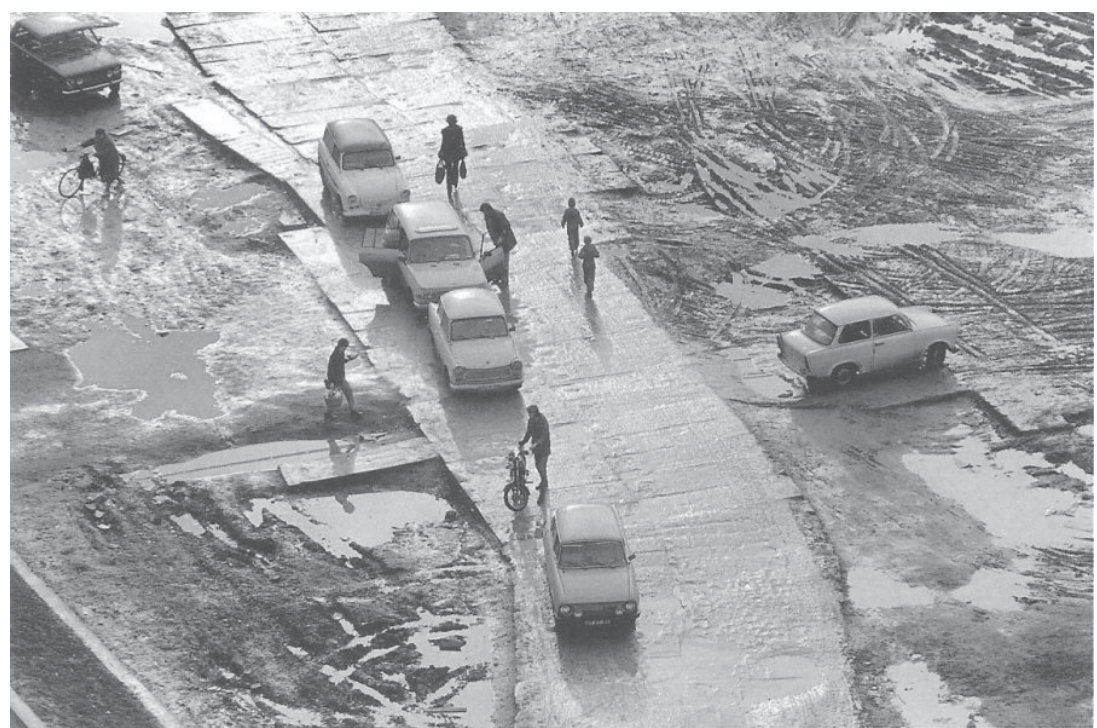

Photo 2 : Grünau à l'époque des Schlammhäuser en 1982

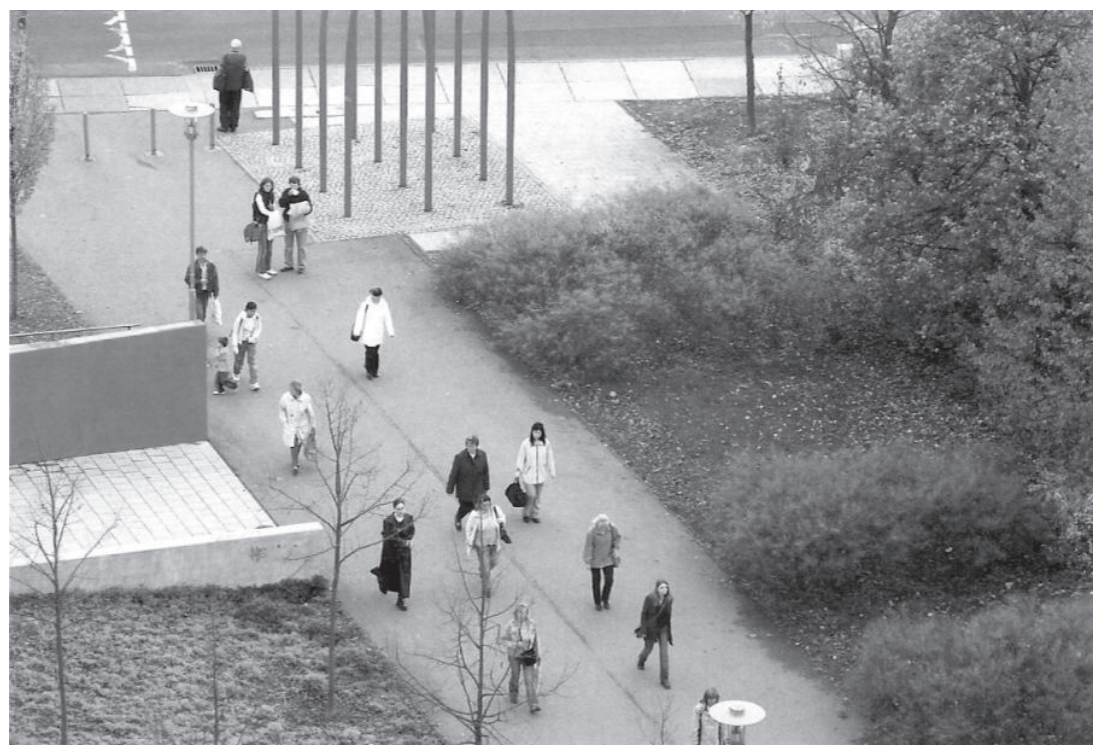

Photo 3 : La même rue en 2005 - Stuttgarter Allee

9 - De 15 à 20\% des enquêtés sont chômeurs ou sont partis en préretraite pour mettre fin aux périodes de non-activité prolongées. Le nombre des bénéficiaires des minima sociaux se situe dans la moyenne de la ville (20\%). Un peu plus d'un tiers des habitants touche moins de $900 €$ par mois, un autre tiers a des revenus compris entre 900 et $1300 €$ par mois, un quart a des revenus approchant les 1500 à 2000 $€$ par mois, et seuls quatre ménages ont des revenus sensiblement supérieurs à $2000 €$ par mois (jusqu'à $80000 €$ par an pour deux cas). Les caractéristiques de ces ménages montrent la forte hétérogénéité sociale de ces immeubles.
I'embellissement qu'il était censé apporter. Par son rôle public, il a été le porte-voix d'une large part des habitants, et a participé de la reprise en main par les habitants et certains bailleurs destinée à construire une image différente des grands ensembles, plus positive.

\section{A l'inverse, une vision positive du quartier}

A l'opposé des stratégies de rénovation menées par le Land ou la mairie, Grün As se fait aussi le porte-voix d'une image positive du quartier. Le journal est ainsi partie prenante de campagnes de marketing pour améliorer l'image du quartier à l'extérieur, et valorise fortement l'évolution du quartier, qui a permis notamment le passage depuis la fin des années 1990 de "Grauau " à Grünau, à savoir la transition de l'époque originelle des grands ensembles entourés de boue (les Schlammhäuser) à un quartier verdi et verdoyant où I'aménagement des espaces publics offre un environnement hautement plus valorisé (photos 2 et 3$)$. Cette vision positive reflète sensiblement les perceptions des habitants sur l'évolution du quartier dans le contexte du déclin et leur attache- ment résidentiel aux Plattenbauten.

\section{L'attachement résidentiel : vivre en Plattenbauten à Leipzig}

La construction interne positive visant à combattre les spirales du déclin s'exprime non seulement à travers un projet journalistique, mais se retrouve également dans les représentations sociales construites par les habitants eux-mêmes. A la différence des enquêtes quantitatives par questionnaires de Schulz à Berlin Marzahn (2004) ou Bernt et Kabisch à Leipzig Grünau (2006), nous avons adopté une approche purement qualitative par entretiens semi-directifs avec une quarantaine d'habitants des grands ensembles, représentatifs de l'ensemble des catégories sociales ${ }^{9}$. Ces entretiens ont fait émerger un processus quasi unanimement partagé par les personnes rencontrées, celui d'un attachement résidentiel extrêmement ancré. Cet attachement repose sur quatre piliers qui font la trame des récits compilés.

\section{Une entrée dans le confort moderne, et une mo- dernité non démentie}

Pour la plupart des habitants rencontrés, l'installation dans les grands ensembles a correspondu à l'entrée dans une forme de confort moderne (Bernt et Kabisch, 2005), matérialisée par un niveau d'équipements avancé, la présence de salles d'eau et sanitaires à l'intérieur de l'appartement, une meilleure isolation et un agrandissement de la surface disponible par personne. Pour les plus anciens, l'obtention d'un appartement en temps de crise du logement était souvent un long parcours du combattant de plusieurs années, et les Plattenbauten ont pu constituer une bouffée $d^{\prime}$ air pour obtenir une première forme d'autonomie et de confort.

Ce confort moderne a contribué grandement à l'attachement des habitants à leur lieu de vie Encadré 2 :

" En obtenant cet appartement ici, nous avons fait partie des gens les plus heureux et chanceux de la RDA " (L. et M., 65 et 70 ans, ont emménagé dans leur appartement en 1973, au chômage avant la retraite).

"Pour nous, ici, c'est la Heimat", à savoir une sorte de patrie intérieure, terme parmi les plus forts pour exprimer l'attachement personnel à un lieu (B., 63 ans, institutrice, a emménagé en 1974).

attachement non démenti par la patine du temps et l'évolution des standards. L'attachement peut même se transformer en panégyrique, puisque certains sont même allés jusqu'à comparer la valeur architecturale des Plattenbauten avec I'héritage du mouvement du Bauhaus.

Cette modernité continuée par d'autres moyens 
se retrouve peu ou prou dans tous les récits, insistant sur la qualité de la construction, à l'opposé des clichés traditionnels sur ce type d'habitat. La qualité des Plattenbauten est alors comme un secret qu'on garde de l'intérieur : les connaisseurs savent, les sceptiques critiquent et sont souvent surpris du contraste entre l'image qu'ils s'en font et la réalité de ce qu'ils voient. C'est ce cadre reverdi et moderne qui explique en grande partie le souhait quasi unanime de rester le plus longtemps possible dans son appartement.

\section{Encadré 3 :}

"Le quartier a mauvaise réputation, notamment pour la propreté, mais c'est ici bien plus propre que dans de nombreux autres quartiers. (...) Mais quand les gens viennent ici, ils sont surtout étonnés de voir à quel point c'est agréable c'est plus l'effet " aha! " et la bonne surprise que les critiques. (...) Ici, il y a de la place, beaucoup de place, et on peut tout utiliser. II y a plein d'avantages, comme le câble pour Internet, le chauffage. Et maintenant, ils sont en train de construire l'ascenseur " (W. et L., 31 et 36 ans, Leipzigois revenus à Grünau après un passage par le centre-ville).

\section{I'aboutissement du parcours résidentiel}

Les conditions de confort, de loyer et d'environnement sont suffisamment attractives pour convaincre tous les types d'habitants de rester, des plus socialement défavorisés à ceux vivant dans le plus grand confort.

\section{Encadré 4 :}

"Ici, j'y reste jusqu'à l'extrême onction. Je n'al pas besoin de m'en occuper. Pourquoi voudrais-je déménager alors que tout va bien ? " (U., 60 ans, veuve, première occupante de son appartement, présente depuis 1977, Mockau)

La Platte est ainsi envisagée comme l'aboutissement d'un parcours résidentiel, et non comme une simple phase transitoire dans l'attente de trouver un logement de standing plus élevé. Cela rappelle que, aujourd'hui encore, les Plattenbauten ne sont pas strictement des logements sociaux dans I'Est de I'Allemagne, car ils étaient des logements pour la classe supérieure du monde ouvrier, représentant une sorte de promotion sociale, à l'opposé de la représentation des tours et barres de logements sociaux qu'on peut trouver à l'Ouest de I'Allemagne ou en France (Fourcaut et Dufaux, 2004). Cet attachement s'explique notamment par les avantages des réseaux de proximité que les grands ensembles procurent.

\section{l'avantage des réseaux de proximité}

Le troisième pilier de l'attachement résidentiel se retrouve à la confluence des réseaux de sociabilité. Même si beaucoup regrettent une certaine dis- solution des liens sociaux par rapport à la période précédant la chute du Mur, et la disparition progressive des communautés d'immeubles (Keller, 2007), les réseaux de sociabilité se tissent au sein du quartier, de la coopérative de logement et dans les grands ensembles en général.

\section{Encadré 5 :}

" Le WK8 est le meilleur pour cela : ici, il y a un vrai sentiment de communauté. Ici, c'est comme au village, on sait où sont les enfants, on sait que les autres veillent. C'est comme une communauté villageoise. II y avait une communauté d'immeuble ici, mais que nous avons organisée nous-mêmes, ce n'était pas les communautés officielles. Après la Wende, naturellement, cela a diminué, mais cela revient peu à peu " (D. et $\mathrm{P}$., 60 et 65 ans, diplômes d'ingénieur mais taxi pour I'une depuis la Wende, et restaurateur en préretraite pour l'autre).

Des communautés d'immeubles (Hausgemeinschaft) se sont ainsi (re)créées, donnant un caractère quasi identitaire à certains ensembles. L'image mobilisée est alors celle du village. Ce sentiment de communauté est suffisamment fort pour être préféré à I'horizon de la propriété individuelle.

\section{le refus de devenir propriétaire}

Le refus de devenir propriétaire d'une maison n'a d'égal que l'attachement aux grands ensembles. Certains habitants sont de toute façon dans I'incapacité financière de le devenir, mais les plus stables économiquement ne le souhaitent pas davantage. Ce refus correspond en fait aussi à la structure de propriété générale à l'Est de I'Allemagne, où les propriétaires occupants sont très peu nombreux, sensiblement moins que dans la partie occidentale (Haller, 2002) ${ }^{10}$.

\section{Encadré 6 :}

"Moi, propriétaire ? Je n'y ai jamais songé, j'avais envie d'avoir mon calme ici. Pas envie de me charger avec d'autres encombrements dans une maison, comme de faire le ménage constam ment " (N. et P., 62 et 64 ans, maître de conférences à l'université et dirigeant d'une concession, installés depuis 1977, Mockau).

Cet attachement contraste à la fois avec un certain discours public sur les grands ensembles et les effets des politiques publiques de rénovation comme Stadtumbau Ost, dont la critique se comprend d'autant mieux à I'aune de l'attachement résidentiel manifesté par les habitants des Plattenbauten. On pourrait presque y lire les contours d'un 'effet Plattenbau' inversé (Florentin, 2009) : les lieux les plus décriés de l'extérieur sont ceux où l'attachement résidentiel se montrerait parmi les plus intenses, en réaction. Pour au-
10 - De manière générale, I'Allemagne tout entière a un taux de propriétaires occupants beaucoup plus bas que le reste de l'Europe $145 \%$, contre $55 \%$ en France ou $70 \%$ au Royaume-Uni). 
tant, un certain nombre d'impondérables viennent nuancer ce tableau idyllique.

\section{LES LIMITES À APPORTER À L'ALLÉGORIE DES PLATTEN : LES DIFFICILES DÉFIS À RELEVER POUR CES QUARTIERS MAL CONSIDÉRÉS}

Deux éléments permettent de nuancer malgré tout I'unanimité de cette image interne extrêmement positive des grands ensembles : I'effet " coopérative " et le vieillissement prononcé des habitants des grands ensembles.

\section{L'effet coopérative ?}

La très grande part des habitants interrogés appartenait à la coopérative de logement Kontakt, très active dans la lutte contre les processus de déclin et l'adaptation aux évolutions du marché immobilier que ces phénomènes ont engendrées (Baron et al., 2010). Le rôle de la coopérative dans le quotidien des habitants, et notamment I'offre de services à la personne nombreux et souvent gratuits, entretiennent un sentiment d'attache forte au quartier, et permettent de recréer un sentiment d'identité puissant, comparable au projet initial de la création des grands ensembles (Hannemann, 1995). Même si les habitants rencontrés extérieurs à la coopérative partageaient globalement les mêmes perceptions et pratiques sociales que leurs homologues vivant dans les immeubles de la coopérative Kontakt, un biais lié à l'affiliation des membres de l'échantillon n'est pas à exclure. Au fond, cela souligne peut-être l'influence de la représentation sociale des habitants sur la politique volontariste du bailleur coopératif, l'ayant poussé à refuser la politique de démolition stricte.

Cependant, comme le soulignent Carsten Keller (2007) et Marlies Schulz (2004), une partie des Plattenbauten subit objectivement un mouvement de paupérisation et on observe ailleurs en Allemagne un mouvement d'opposition entre les habitants anciens des grands ensembles et les nouveaux arrivants parfois plus paupérisés (Kil, 2004). Les habitants de la coopérative ont pu parfaitement construire une forme d'entre-soi, créant une communauté symbolique de référence. $L^{\prime}$ argument de la communauté installe une position symbolique, qui permet à son tour de se distancer de ceux que l'on considère comme " asociaux" .

"L'antichambre d'un futur hospice pour personnes âgées " $(0$. , femme seule, 60 ans, allocataire des minima sociaux, habitant le quartier depuis 1979)

Au-delà des éventuels biais liés à la position de la coopérative, une tendance profonde permet de nuancer l'enthousiasme débordant des habitants des grands ensembles, celle du vieillissement généralisé de la population (carte 2). Sur la carte de la ville, deux ceintures ou couloirs du vieillissement émergent, plus en marge de la ville, dans les zones Ouest et Est de la commune. Certains quartiers comme Heiterblick, Mockau ou Grünau, trois quartiers principalement de grands ensembles, ont ainsi vu leur population vieillir en moyenne de dix à quinze ans dans un laps de temps d'à peine seize ans, ce qui montre la force du vieillissement qui les a touchés. Ce vieillissement accéléré permet ainsi de souligner un certain manque de mixité intergénérationnelle au sein de ces quartiers de grands ensembles, dont la pérennité est du même coup moins assurée à moyen ou long terme sans un retournement et des mouvements migratoires et des perceptions dont ils font l'objet.

\section{Encadré 7 :}

" Je trouve ça vraiment triste : les jeunes ne restent plus ici, ou alors ne sont là que pour peu de temps. Ce sont surtout des personnes âgées. La jeunesse part dans les anciens Länder, parce qu'il n'y a plus de travail ici. Avant, tout le monde vivait ici, on avait aussi des gens en fauteuil roulant. C'était beaucoup plus mélangé. C'est quand même mieux quand il y a plusieurs générations qui vivent ensemble. Les jeunes sont partis du quartier après la Wende, à la recherche d'un travail. Toutes les entreprises ont fermé. Et il n'y avait donc plus de possibilités d'avoir des formations professionnelles. Depuis des années, ici, c'est devenu un hospice pour personnes âgées. Avant, on disait ici que c'était une ville dortoir, mais maintenant, c'est un hospice, car il n'y a plus d'industrie, les gens ne vont plus travailler" $(0$. ., femme seule, 60 ans, allocataire des minima sociaux, habitant le quartier depuis 1979 , Grünau)

Ce constat de quartiers vieillissants est fait par les habitants eux-mêmes, souvent lucides sur la situation d'un quartier auxquels ils sont si attachés (photos 4 et 5). Les spirales du déclin viennent ici renforcer les tendances au vieillissement, et ne sont pas contrebalancées par les qualités du quartier défendues par ses propres habitants.

\section{CONCLUSION}

Souvent décriés par les discours publics et par le stigmate idéologique qui les associe à la période socialiste, les grands ensembles de l'Est de I'Allemagne traversent une phase contradictoire. IIs sont non seulement l'objet de discours publics négatifs, mais également la cible des politiques de démolition de la rénovation urbaine, ce qui crée et amplifie dans le même temps l'expression d'un attachement presque sans faille de la part de leurs habitants. Pour autant, une tendance récente à la paupérisation et au vieillissement prononcé en font un laboratoire des effets du déclin et de la transition post-socialiste, peut-être annonciateur 


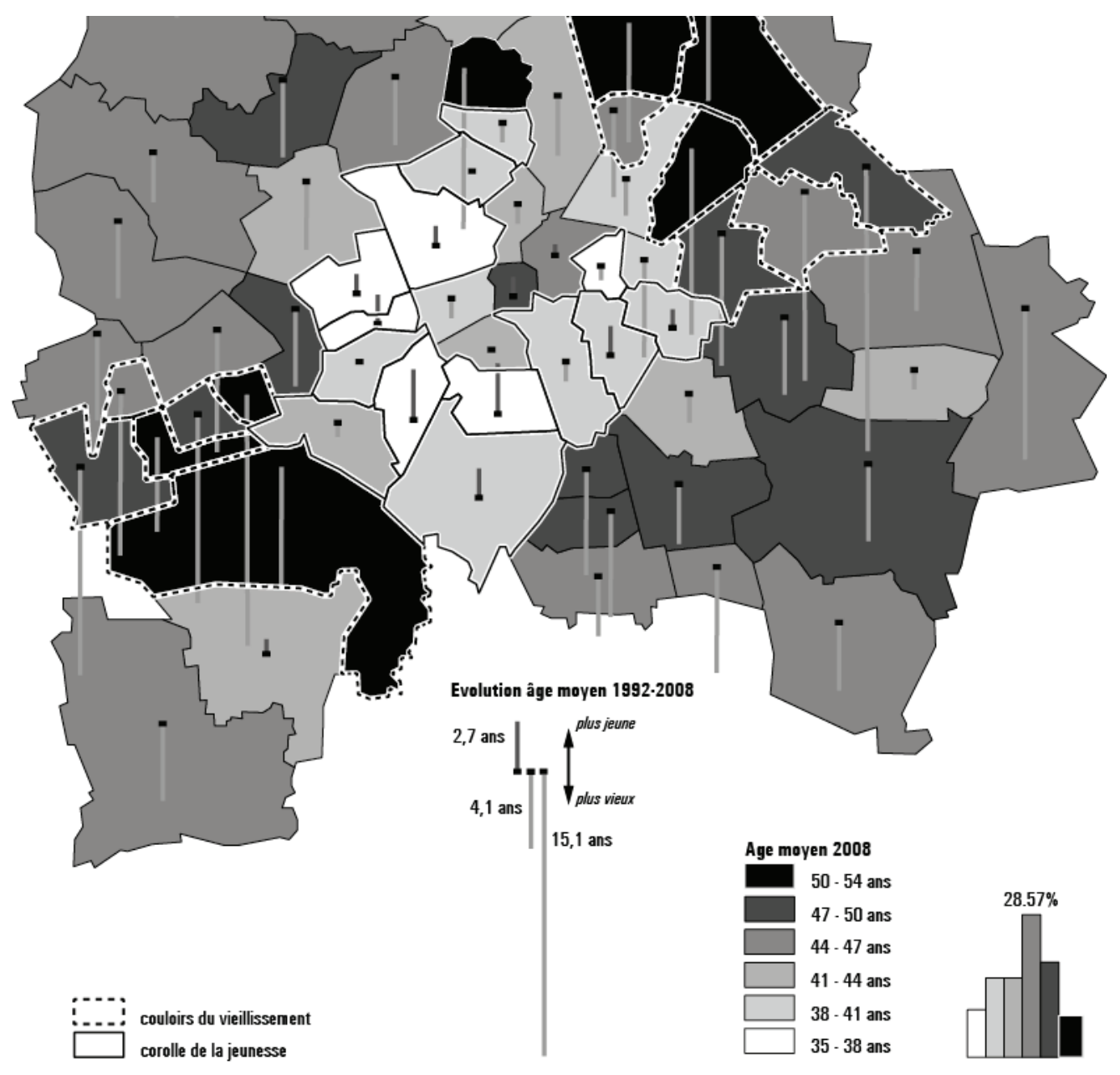

Carte 2: Leipzig et le tournant démographique (1992-2008)

des évolutions que pourraient connaître l'ensemble des villes concernées par ce processus.

"L'effet Plattenbau ", quoique combattu par les perceptions d'une population vieillissante mais enthousiaste pour l'avenir de ses quartiers, semble bien prégnant. Les politiques urbaines de rénovation urbaine n'ont jusqu'à présent fait qu'amplifier cette dynamique régressive et confirmer le vieillissement moyen des grands ensembles. Elles ont finalement accru des processus de perforation urbaine.

Face à cette tendance lourde, certains acteurs du monde immobilier cherchent malgré tout à réinventer une forme de modernité des grands ensembles (Baron et al., 2010), pour traverser et dépasser une période de triple rejet, urbain, symbolique et idéologique, assez prononcé. II est sans doute encore trop tôt pour savoir si ce regain de modernité des grands ensembles peut rencontrer un quelconque succès.

\section{BIBLIOGRAPHIE}

ACT CONSULTANTS, BAUHAUS UNIVERSITÄT WEIMAR, 2007, Les politiques de renouvellement urbain des villes d'Europe centrale illustrées par la réhabilitation des quartiers existants. La ville de Leipzig en Allemagne, Paris, ANAH et CNDC.

ARNHOLD T., mars 2005, Die Platte ist besser als ihr Ruf, Helmholtz-UfZ.
BAFOIL F., 2006, Transfert institutionnel et européanisation. Une comparaison des cas est-allemand et est-européens, Revue Internationale de Politique Comparée, 13 (2), p. 213-238.

BARON M., CUNNINGHAM SABOT E., GRASLAND C., RIVIĖRE D., VAN HAMME G. (dir), 2010, Villes et régions en décroissance : maintenir la cohésion territoriale, Hermès Lavoisier, $346 \mathrm{p}$.

BERNT M., 2003, Risiken und Nebenwirkungen des Stadtumbaus. www.schrumpfende-stadt.de.

BERNT M., 2005, Die politische Steuerung des Stadtumbaus in Leipzig-Grünau, UFZ Diskussionpapiere.

BERNT M., 2009, Partnership for Demolition: The Governance of Urban Renewal in East Germany's shrinking cities, IJURR, 33-3, p. 754-769.

BERNT M. \& FRITSCHE M., 2008, Kooperation -mit wem? Bewohnerbeteiligung im Stadtumbau Ost, in VETTER A. (Hrsg.) Erfolgsbedingungen lokaler Bürgerbeteiligung Städte und Regionen in Europa 16 VS Verlag für Sozialwissenschaften, Wiesbaden, p. $237-258$.

BERNT M. \& KABISCH S., 2006, Ostdeutsche Grosswohnsiedlungen zwischen Stabilisierung und Niedergang. dis $P(164)$, p. 5-15. 


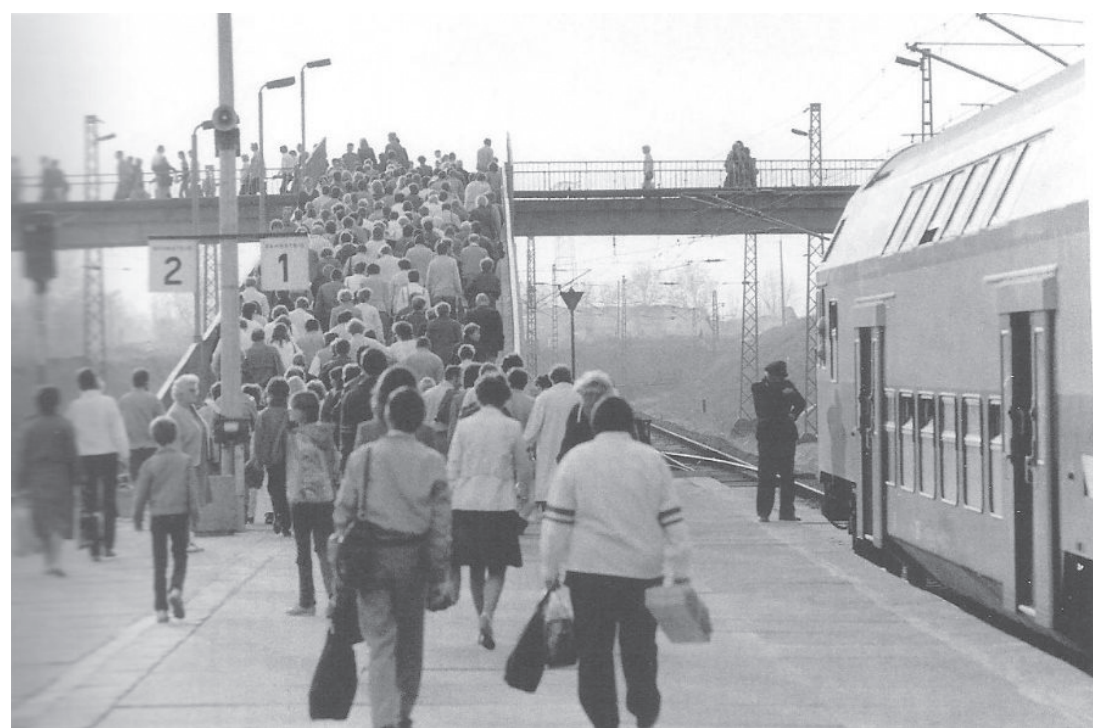

Photo 4 : La station de S-Bahn de Grünau en 1985

Photo 5 : La station de S-Bahn de Grünau en 2005

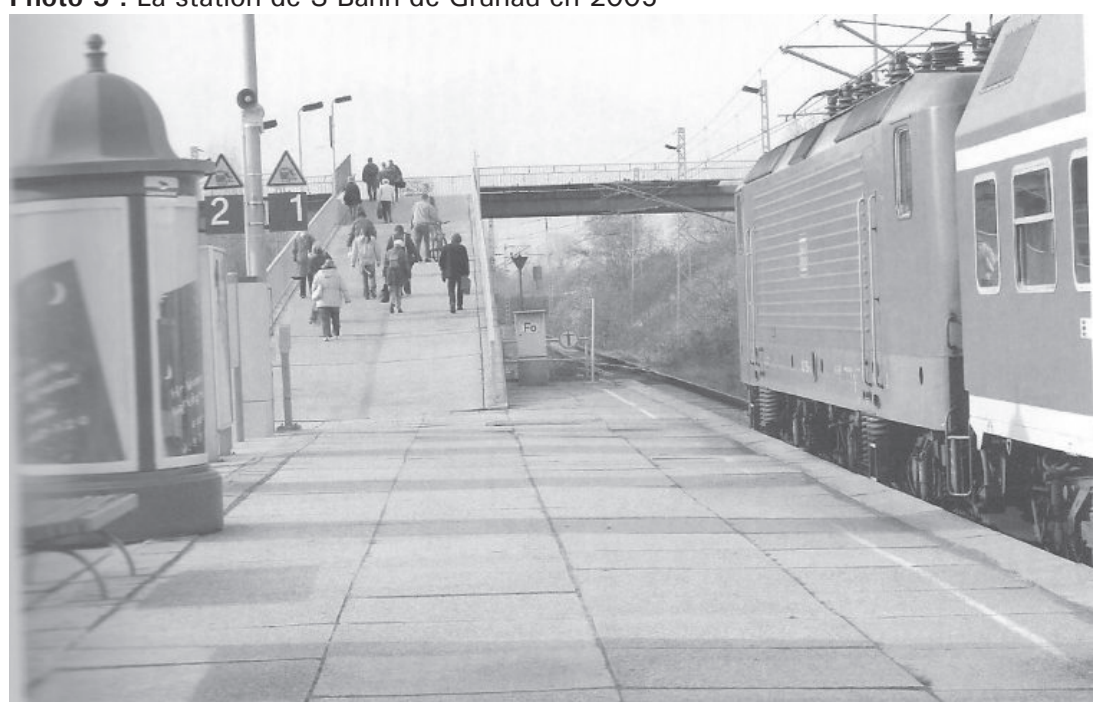

BERNT M., KABISCH S. \& PETER A., 2005, Die Auswirkungen von Schrumpfung und Stadtumbau auf die Stadtgesellschaft: der Fall Weisswasser, Berichte zur deutschen Landeskunde, Heft 79-1, p. 33-57.

BERNT M., RÖSSLER S. \& KABISCH S., 2005, Interessensgegensätze erfordern neue Umsetungsstrategien. Freiraumentwicklung und Stadtumbau in der Grosssiedlung Leipzig-Grünau, IÖR, 54-9, p. 15-20.

BONTJE M., 2004, Facing the challenge of shrinking cities in East Germany: the case of Leipzig, Geojournal (61), p. 13-21.

BRANDSTETTER B., LANG T. \& PFEIFER A., 2005, Umgang mit der schrumpfenden Stadt - ein Debattenüberblick, Berliner Debatte Initial, Heft 6, p. 55-68.

COMMISSION EUROPÉENNE, 2005, Angesichts des demographischen Wandels - eine neue Solidarität zwischen den Generationen, Livre vert.

FOL S. \& CUNNINGHAM-SABOT E., 2010, "Déclin urbain " et Shrinking Cities : une évaluation critique des approches de la décrois- sance urbaine, Annales de Géographie, $\mathrm{n}^{\circ} 674$, p. 359-383.

FLORENTIN D., 2008, Leipzig ou la ville perforée une "shrinking city" modèle ? Paris, Université de Paris 1 Panthéon Sorbonne, mémoire de $M 1$ sous la direction de S. Fol et F. Dufaux, $130 \mathrm{p}$.

FLORENTIN D., 2009, Le déclin au quotidien. Stratégies immobilières et pratiques sociales face au déclin à Leipzig, Paris, Université de Paris 1 Panthéon Sorbonne, mémoire de M2 sous la direction de S. Fol et F. Dufaux, 204 p.

FLORENTIN D., FOL S. \& ROTH H., 2009, La "Stadtschrumpfung " ou "rétrécissement urbain " en Allemagne : un champ de recherche émergent, Cybergéo (445). http://www.cybergeo.eu/index22123.html

FOURCAUT A. \& DUFAUX F. (dir), 2004, Le monde des grands ensembles, Creaphis, 256 p.

FRITZSCHE A., 2006, Die Grosswohnsiedlung aus Bewohnerperspektive. Intervallstudie "Wohnen und Leben im Grünau", Neue Stadtlandschaften im Zeichen der Schrumpfung, UfZ.

GERMES M., BRAILICH A., SCHIRMEL $H_{\text {., }}$ GLASZE G. \& PÜTZ R., 2010, Les grands ensembles de banlieue comme menaces urbaines ? Discours comparés - Allemagne, France, Pologne, Annales de Géographie, nº675, p. 515-535.

GLOCK B. \& HÄUSSERMANN H., 2004, New Trends in Urban Development and Public Policy in Eastern Germany: Dealing with the vacant Housing Problem at the Local Level, IJURR, vol 28.4 .

GUIHÉRY L. \& WERNER J., 2005, Les finances publiques en Allemagne. Quelles réformes? Revue de I'OFCE, 94, p. 111-137.

HALLER C., 2002, Leerstand im Plattenbau, Berlin, Edition Stadt und Region, band 4

HANNEMANN C., 1995, 2004, Architecture as an ideology: industrialization of housing in the GDR, Berlin, Humboldt Universität, Stadt- und Regionalsoziologie, Working Papers Nr. 2 A, 26 p.

HANNEMANN C., 2003, Schrumpfende Städte in Ostdeutschland - Ursachen und Folgen einer Stadtentwicklung ohne Wirtschaftswachstum, Politik und Zeitgeschichte B28, p. 16-24.

KABISCH S., FRITZSCHE A. \& BERNT M., 2006, Die Grosswohnsiedlung aus Bewohnerperspektive - Intervallstudie "Wohnen und Leben im Grünau", Neue Stadtlandschaften im Zeichen der Schrumpfung, UfZ. 
KABISCH S., BERNT M. \& FRITZSCHE A., 2005, Intervallstudie "Wohnen und Leben im Grünau", Stadt Leipzig, Amt für Statistik und Wahlen, Statistischer Quartalsbericht 1, p. 12-15.

KABISCH S., PETER A. \& BERNT M., 2007, Stadtumbau Ost aus der Sicht der BewohnerWahrnehmungen, Erwartungen, Partizipationschancen, dargestellt anhand von Fallbeispielen. Information zur Raumentwicklung, Heft 1, p. 37-47.

KELLER C., 2007, La population des grands ensembles de l'ex-RDA. Fractionnement social et luttes pour la respectabilité, Espaces et sociétés, $n^{0} 128-129$, p. 87-101.

KIL W., 2004, Testfall für den Epochenwandel - der "Stadtumbau Ost" beseitigt Wohnungen, ohne nach dem Kern des Problems zu fragen. www.schrumpfende-stadt.de .

KIL W., 2001, Überflüssige Städte: im Osten wird der Leerstand bekämpft, doch der Wohnungsmarkt ist nicht das Problem, Deutsche Bauzeitung.

KOMMISSION LEHMANN-GRUBE, 2000, Wohnungswirtschaftlicher Strukturwandel in den neuen Bundesländern, BVBW.

KUNZE C. \& URBANIAK N., 2002, Veränderung des Wirtschaftsstandorts Leipzig 1995-1999, in KUNZE C., Grossstädte im Transformationsprozess vor dem Hintergrund europäischer Integration und internationalen Strukturwandels. Leipzig und Wroclaw 1995 bis 1999 im Vergleich. Universität Leipzig - Transformation.

LÜTKE DALDRUP E., 2003, Die perforierte Stadt - neue Räume im Leipziger Osten, Information zur Raumentwicklung, cahier 1 .

NOYÉ C. \& LELÉVRIER C., 2009, Diversification de l'habitat. Diversification fonctionnelle dans les opérations de rénovation urbaine en lle de France, DREIF.

NUISSL H. \& RINK D., 2004, Sprawl und Schrumpfung: das Beispiel Leipzig, UFZ-Diskusssionspapiere.

NUISSL H. \& RINK D., 2006, Suburbanisierung in schrumpfenden Stadtregionen: ein neuer Stadttypus? Leipzig und Liverpool im Vergleich, Neue Stadtlandschaften im Zeichen der Schrumpfung, UfZ.
NUISSL H. \& RINK D., 2005, The production of urban sprawl in eastern Germany as a phenomenon of post-socialist transition, Cities 22 (2), p. 123 - 134 (Elsevier).

PRETECEILLE E., 2004, Définir et analyser la ségrégation sociale, in Les mécanismes fonciers de la ségrégation, ADEF Paris, p. 9-29.

SCHMIDT H., 1959, Industrialisierung und Städtebau, Beiträge zur Architektur 1924-1964.

SCHÖNFELDER M.-K. \& KIRSCHNER H., 2006, Grünau Fotolesebuch, Pro Leipzig.

SCHULZ M., 2004, Stadtumbau Ost - Berlin Marzahn, Projektseminar, Sommersemester 2003.

STEINFÜHRER A., 2006, Städtische Images und ihre Bedeutung im Stadtumbau - Beispiel Johanngeorgenstadt, Neue Stadtlandschaften im Zeichen der Schrumpfung, UfZ.

STEINFÜHRER A. \& KABISCH S., 2005, Images einer langfristig schrumpfende Stadt. Das Beispiel Johanngeorgenstadt (Sachsen), Berichte zur deutschen Landeskunde, 79/1, p. 5-31.

WIECHMANN T. \& SIEDENTOP S., 2005, Monitoring des Stadtumbaus - eine neue Herausforderung? Flächemanagement und Bodenordnung, ${ }^{\circ} 5$, p. 206-214.

WIECHMANN T., 2007, Conversion strategies under uncertainty in Post-socialist Shrinking Cities: The example of Dresden in Eastern Germany, The Future of Shrinking Cities -Problems, Patterns and Strategies of Urban Transformation in a Global Context, Berkeley, Institute for urban and regional development, monographs, p.5-16.

WIESSNER R., 2007, Einkommendifferenzierung und weitere Differenzierung der sozialen Schichten und Gruppen, in KUNZE C. et LENK T., Grossstädte im Transformationsprozess vor dem Hintergrund europäischer Integration und internationalen Strukturwandels. Leipzig und Wroclaw 2000 bis 2004 im Vergleich, Leipzig, Transformation - Leipziger Beiträge zur Wirtschaft und Gesellschaft n²1-22.
Adresse de I'auteur :

Ecole Normale Supérieure, UMR 8504 Géographie-cités Daniel.Florentin@ens.fr 